\title{
Episodic memory and speed/attention deficits are associated with Alzheimer-typical CSF abnormalities in MCI
}

\author{
ARTO NORDLUND, SINDRE ROLSTAD, OLA KLANG, KARIN LIND, MONA PEDERSEN, \\ KAJ BLENNOW, ÅKE EDMAN, STEFAN HANSEN, AND ANDERS WALLIN \\ Institute of Neuroscience and Physiology, Sahlgrenska Academy at Göteborg University, Mölndal, Sweden \\ Department of Psychology, Göteborg University, Göteborg, Sweden
}

(Received July 7, 2007; Final Revision March 19, 2008; Accepted March 21, 2008)

\begin{abstract}
Mild cognitive impairment (MCI) is regarded as the prodromal stage of dementia disorders, such as Alzheimer's disease (AD).

Objective: To compare the neuropsychological profiles of MCI subjects with normal concentrations of total tau (T- $\tau$ ) and $\mathrm{A} \beta 42$ in CSF (MCI-norm) to MCI subjects with deviating concentrations of the biomarkers (MCI-dev). MCI-norm $(N=73)$ and MCI-dev $(N=73)$ subjects were compared to normal controls $(N=50)$ on tests of speed/attention, memory, visuospatial function, language and executive function.

Results: MCI-norm performed overall better than MCI-dev, specifically on tests of speed and attention and episodic memory. When MCI-dev subjects were subclassified into those with only high T-tau (MCI-tau), only low $\mathrm{A} \beta 42$ (MCI-A $\beta$ ) and both high T-tau and low A $\beta 42$ (MCI-tauA $\beta$ ), MCI-tauA $\beta$ tended to perform slightly worse. MCI-tau and MCI-A $\beta$ performed quite similarly.

Conclusions: Considering the neuropsychological differences, many MCI-norm probably had more benign forms of MCI, or early non-AD forms of neurodegenerative disorders. Although most MCI-dev performed clearly worse than MCI-norm on the neuropsychological battery, some did not show any deficits when compared to age norms. A combination of CSF analyses and neuropsychology could be a step toward a more exact diagnosis of MCI as prodromal AD. (JINS, 2008, 14, 582-590.)
\end{abstract}

Keywords: Mild cognitive impairment, Neuropsychology, Cognition, Total tau, A $\beta 42$, AD

\section{INTRODUCTION}

Alzheimer's disease (AD) is the most common form of dementia. The clinical tradition has been that AD cannot be diagnosed until dementia is present. In recent years, however, interest in identifying dementia disorders early in the course of the disease has increased. In these efforts the concept of mild cognitive impairment (MCI) has attracted attention and become the target of a number of studies.(Grundman et al., 2004; Morris et al., 2001; Petersen et al., 1999; Ritchie et al., 2001). MCI is conceptualized as the boundary or transitional state between normal brain aging

Correspondence and reprint requests to: Arto Nordlund, Institute of Neuroscience and Physiology, Sahlgrenska Academy, Wallinsgatan 6, SE 43141 Mölndal, Sweden. E-mail: arto.nordlund@neuro.gu.se and dementia. In most studies MCI has been considered the prodromal stage of $\mathrm{AD}$, and consequently characterized by memory impairment (Morris et al., 2001; Petersen, 2000; Storandt et al., 2002). The conversion rate of MCI to AD has been reported to be $10-15 \%$ per year (Morris et al., 2001; Petersen, 2000). There have, however, also been reports suggesting that $\mathrm{MCI}$ is a heterogeneous condition in which several types of cognitive impairment is present, memory impairment not necessarily being the most dominant characteristic (Busse et al., 2006; Nordlund et al., 2005). According to the most recent recommendations, MCI criteria include the following: (i) the person is neither normal nor demented; (ii) there is evidence of cognitive deterioration both objectively and subjectively, and (iii) activities of daily living are preserved and complex instrumental functions are either intact or minimally impaired (Winblad et al., 
2004). There is as of yet no generally accepted method to determine if a patient with MCI has incipient AD (i.e. will progress to $\mathrm{AD}$ with dementia, or have a benign form of MCI without progression).

The neuropsychological profile of incipient $\mathrm{AD}$ (i.e., the form of MCI preceding AD with dementia) has been described in a number of studies (Bozoki et al., 2001; Morris et al., 2001; Petersen, 2000; Petersen et al., 1999). Although the focus of these studies has been memory impairment, mild impairment in other cognitive domains also has been reported (Bozoki et al., 2001; Petersen et al., 1999; Ritchie et al., 2001). According to some studies, the risk of $\mathrm{AD}$ is significantly increased when multiple cognitive domains are impaired (Bozoki et al., 2001; Guarch et al., 2004; Rasquin et al., 2004). In these studies, subjects with memory impairment alone were few and progressed to dementia at a slower rate than did subjects with other types of cognitive impairment. Some studies have come to differing conclusions. According to one, patients at highest risk for $\mathrm{AD}$ were those who in addition to memory impairment showed deficits in the language (naming) domain (Blackwell et al., 2004), whereas another study found that naming tests were not useful when diagnosing MCI and AD (Testa et al., 2004). In another study the conclusion was that patients with executive impairment were at highest risk for AD (Albert et al., 2001). Comparing studies with different patient samples and neuropsychological batteries gives a slightly contradictory impression, which emphasizes the importance of a comprehensive neuropsychological examination when assessing individuals at risk for AD.

Because AD is restricted to the brain, the cerebrospinal fluid (CSF) is an obvious source for biochemical markers for AD. The CSF is in direct contact with the extracellular space of the brain; hence biochemical changes in the brain affect the composition of CSF. Biochemical markers should reflect the central pathogenetic processes, (e.g., the neuronal degeneration and the increased number of plaques and tangles). Since 1995, two CSF biochemical markers for AD have emerged, total-tau (T-tau) and amyloid- $\beta 42$ (A $\beta 42)$ (Andreasen et al., 2003). Tau protein is located in the neuronal axons and the concentration of T-tau in the CSF probably reflects the intensity of neuronal degeneration in chronic neurodegenerative disorders (Blennow, 2004b). A $\beta 42$ is the major component of senile plaques. The decreased level of $\mathrm{A} \beta 42$ in the CSF in AD may be caused by deposition of $\mathrm{A} \beta 42$ in plaques, with lower levels being transported to CSF (Blennow, 2004a). In the last few years increased levels of tau and decreased levels of $\mathrm{A} \beta 42$ have been used to predict AD in MCI subjects with some success (Andreasen et al., 2003; Hansson et al., 2006; Ivanoiu \& Sindic, 2005). One conceivable way of increasing the prognostic specificity of MCI as a preliminary stage of $\mathrm{AD}$ is to link findings in CSF to the neuropsychological profiles in MCI. In two studies on MCI, the relation between CSF biomarkers and neuropsychological findings was examined (Ivanoiu \& Sindic, 2005; Schoonenboom et al., 2005). Both studies found elevated T-tau concentrations primarily to be associated with poor performance on episodic memory tests, whereas decreased $\mathrm{A} \beta 42$ concentrations were associated with poorer general neuropsychological performance. The objective of this study was to compare the neuropsychological profiles of MCI subjects with normal concentrations of total tau and $\mathrm{A} \beta 42$ in CSF to MCI subjects with increased and decreased concentrations of these biomarkers.

\section{MATERIALS AND METHODS}

\section{Subjects and Diagnostic Procedure}

The study was approved by the local ethics committee. Between May 2000 and December 2005, 330 subjects were included in the Göteborg MCI study. The distribution of diagnoses and further subclassifications are illustrated in Fig. 1. The majority (about 3/4) of the subjects was referred by their general practitioners or a specialist to our clinic, whereas about $1 / 4$ came to our clinic on their own initiative; they experienced cognitive decline and contacted our clinic for an examination. The distribution of diagnoses was as follows: MCI $69 \%$, mild AD 16\%, mild vascular dementia ( VaD) $10 \%$, other (unspecified dementia, frontotemporal dementia, and primary progressive aphasia) 5\%. Subjects with major depressive and other severe psychiatric disorders were excluded, whereas subjects with minor depressive symptoms and mild anxiety were not. The diagnosis of MCI was made in congruence with the most recent recommendations (Winblad et al., 2004). MCI was diagnosed by means of medical history and checklists for cognitive symptoms: stepwise comparative status analysis (STEP) for basic cognitive symptoms, cognitive variables 13-20 (memory disturbance; disorientation; reduced abstract thinking; visuospatial disturbance; poverty of language; sensory aphasia; visual agnosia; apraxia) (Wallin et al., 1996), I-Flex, which is a short form of the Executive Interview (EXIT) (Royall et al., 1992), for frontal lobe symptoms (items number-letter task; word fluency; anomalous sentence repetition; interference task; Luria hand sequences; counting task), mini mental status examination (MMSE) (Folstein et al., 1975) and clinical dementia rating (CDR) (Morris, 1997), a global measure of functioning. The information for CDR was gathered from the subject and an informant. For inclusion, subjective and objective (verified by an informant) anamnestic evidence for progressive cognitive impairment for more than 6 months was required. Furthermore, objective cognitive symptoms according to STEP, I-Flex, MMSE and/or CDR were required. Subjects without symptoms according to the checklists were not included, because their cognitive impairment was considered too benign. Neither were subjects with more than two symptoms on STEP and/or a score below 25 on MMSE, because they were considered to fulfil the criteria for dementia.

Out of the 330 subjects included in the study, 228 fulfilled the criteria for a clinical diagnosis of MCI. In order to identify subjects with incipient primary degenerative dementia, usually $\mathrm{AD}$, a tentative diagnosis of primary degenerative $\mathrm{MCI}$ was made at the occurrence of symptoms of MCI, no more 


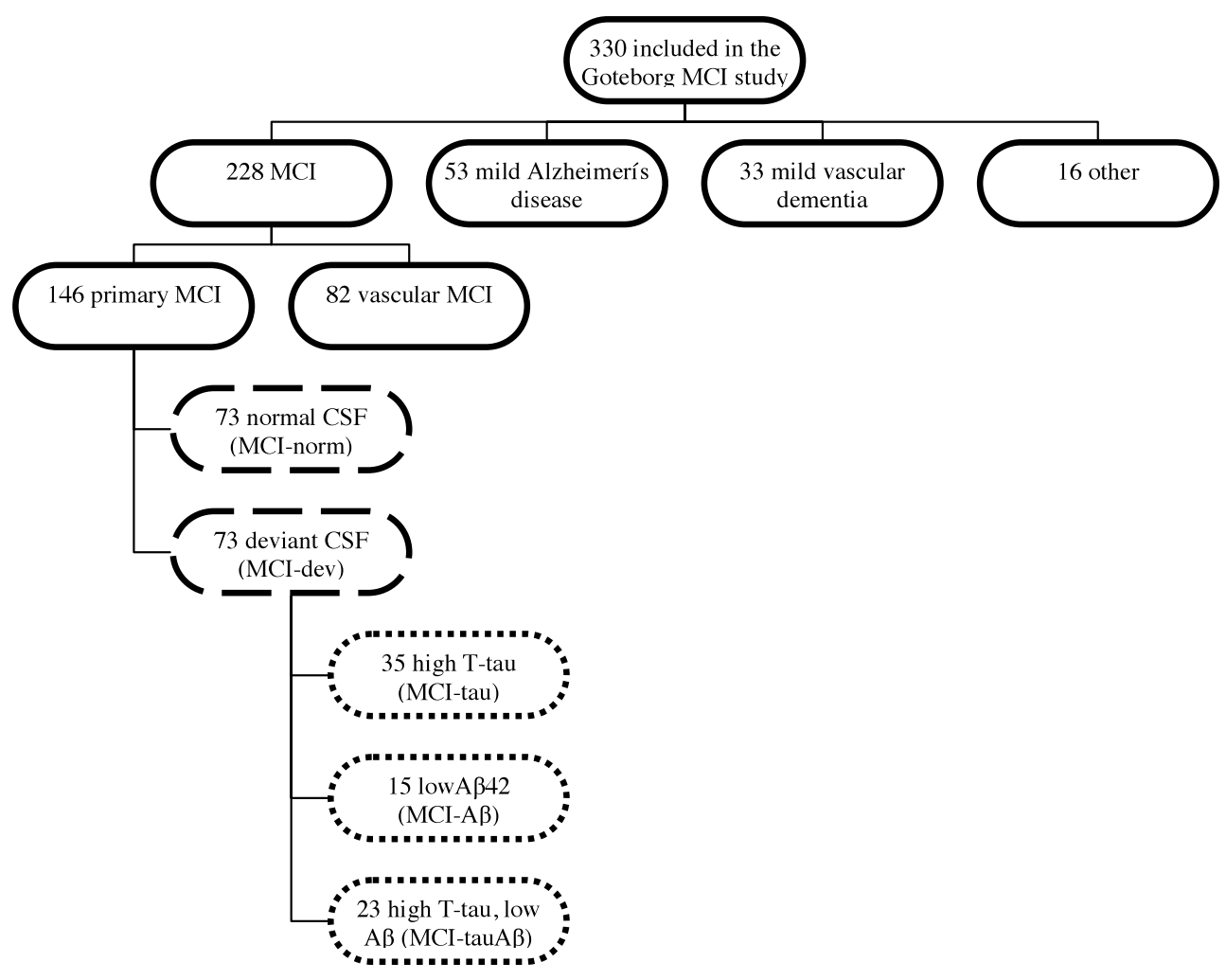

MCI=Mild Cognitive Impairment, $\mathrm{CSF}=$ cerebropinal fluid, $\mathrm{T}$-tau=total tau, $\mathrm{A} \beta=$ amyloid-fl42

Fig. 1. Distribution and subclassification of diagnoses.

than one vascular risk factor without complications and no or insignificant findings on brain imaging. All subjects underwent brain MRI scans, which were evaluated by experienced neuroradiologists. For primary degenerative MCI only insignificant $(=$ mild) white matter changes on a 4-grade scale (Scheltens et al., 1998) and/or few lacunes $(<3)$ or absence of cerebrovascular changes were allowed. Subjects with vascular disease (arterial hypertension, cardiac insufficiency, angina pectoris, cardiac rhythm disturbance, cardiac infarction, TIA, stroke, hyperlipidemia, diabetes mellitus, or peripheral vessel disease) and significant ( $=$ moderate or severe) white matter changes/several lacunes and/or signs of infarctions according to visually assessed brain imaging were considered MCI of vascular aetiology.

Out of the 228 MCI subjects $64 \%$ (146) were considered primary degenerative MCI and $36 \%$ as MCI of vascular aetiology. As the focus of this study is biomarkers associated with $\mathrm{AD}$, the MCI subjects with vascular disease were excluded from the study. For further analyses, the MCI subjects were subclassified according to the subtypes suggested by Petersen (2004): amnestic MCI (isolated memory impairment), multidomain amnestic MCI (memory and other domain[s] impaired), multidomain non-amnestic MCI, and single domain non-memory MCI.

Fifty healthy controls were included in the study. They were mainly recruited from senior citizen organisations and via information meetings on dementia. A few controls were spouses of subjects in the study. Inclusion criteria for controls were that they should be physically and mentally healthy and not experience or exhibit any cognitive impairment. All controls were thoroughly interviewed about their somatic and mental health by a research nurse before inclusion in the study.

\section{Cerebrospinal Fluid Analysis}

CSF samples were collected in polypropylene tubes, and were stored at $-80^{\circ} \mathrm{C}$ pending biochemical analyses, without being thawed and re-frozen. CSF samples were taken at baseline in all MCI cases and controls. CSF T-tau was determined using a sandwich enzyme-linked immunosorbent assay (ELISA) constructed to measure total tau (Blennow et al., 1995). CSF A $\beta 42$ was determined using an ELISA constructed to measure $\mathrm{A} \beta 42$ (Andreasen et al., 1999).

\section{Neuropsychological Assessment Instruments}

Following recommendations by the American Academy of Neurology (AAN) (1996), our neuropsychological examination comprised tests of speed and attention, learning and episodic memory, visuospatial, language and executive functions (Table 1). Within each cognitive domain several aspects of function was assessed, in order to obtain an as complete a picture as possible of the cognitive status of the subjects. 
Table 1. Cognitive domain, specific functions, and neuropsychological tests

\begin{tabular}{|c|c|}
\hline Cognitive domain & Specific functions and neuropsychological tests \\
\hline Speed and attention & Digit Symbol (WAIS-R), Trail making A and B, Attention span/working memory: Digit Span (WAIS-R) \\
\hline Learning and memory & $\begin{array}{l}\text { Verbal episodic memory: RAVLT, Wechsler's Logical Memory (WMS-R), Non-verbal episodic memory: } \\
\text { Rey Complex Figure }\end{array}$ \\
\hline Visuospatial functions & $\begin{array}{l}\text { Perception: Silhouettes (VOSP), Spatial organisation: Rey Complex Figure copy, Construction: Block } \\
\text { Design (WAIS-R) }\end{array}$ \\
\hline Language & $\begin{array}{l}\text { Comprehension: Token Test, subtest V, Comprehension and repetition: ASLD repetition, Confrontation } \\
\text { naming: Boston Naming Test, Abstraction: Similarities (WAIS-R) Word Fluency: FAS }\end{array}$ \\
\hline Executive functions & $\begin{array}{l}\text { Mental control: PaSMO, Divided attention: Dual Task, Planning and inference: WCST-CV64, } \\
\text { Distractibility: Stroop Test, Victoria version, Judgement and calculation: Cognitive Estimation Test }\end{array}$ \\
\hline
\end{tabular}

WAIS-R = Wechler's Adult Intelligence Scale-Revised, RAVLT = Rey Auditory Verbal Learning Test, WMS-R = Wechler's Memory Scale-Revised, VOSP $=$ Visual Object and Space Perception, ASLD = Assessment of Subtle Language Deficits, PaSMO = Parallell Serial Mental Operations, WCSTCV64 = Wisconsin Card Sorting Test-Computer Version 64 (short version).

The neuropsychological test battery presented in Table 1 has been described in detail previously (Nordlund et al., 2005). A few tests may be less well known than the others: the Visual Object and Space Perception (VOSP) Silhouettes sub test consists of 30 silhouettes -15 animals and 15 everyday objects—of increasing difficulty and has been used to assess brain injury in general (Rapport et al., 1998) and also to distinguish mild AD from normal aging (Binetti et al., 1996). The Assessment of Subtle Language Deficits (ASLD) repetition sub test is a test of language comprehension and repetition. It consists of 10 sentences of increasing length and complexity, which the subject is to repeat. In Parallel Serial Mental Operations (PaSMO) the subject is asked to rattle off the alphabet stating the number of the letter after each letter, i.e. A-1-B-2-C-3 . . , and thus it is considered a measure of mental control and tracking.

\section{Neuropsychological Assessment Procedure}

The tests were administered in a standardised sequence and the testing was divided into 2 sessions of 1-2 hours. Verbal tests were varied with nonverbal in each session. The test sequence was also decided on the consideration of risk of contamination on the memory tests. Hence, no test with content, which could affect performance on a memory test, was administered between immediate and delayed recall.

\section{Statistical Analysis}

The comparison of occurrence of APOE4 allele was made with a chi-square test. Several neuropsychological test variables were found to be skewed and were rescaled as appropriate to approximate normality before being entered in the statistical calculations. The data are presented as means \pm standard deviation of the raw data. Demographic data were calculated with ANOVA. Because of differences in age and education between the MCI groups, those variables were entered as covariates in the statistical analyses of neuropsychological data, and group comparisons were made with ANCOVA (SPSS). Multiple comparisons were adjusted for with Sidak correction. In addition, the upper level of false significances was calculated (Eklund \& Seeger, 1965) and expected to be 1,8 , which means that fewer than 2 of the significant tests were the results of coincidence, and that the significant differences are to be considered true. Principal components analysis (PCA; SIMCA-P 10.0) was performed on the data from the test battery (i.e., including all 22 test variables) (Eriksson et al., 2002). Significance of the model was determined by cross validation. The PCA resulted in one significant latent variable that summarized the constituent neuropsychological test variables. The composite score of each subject is considered to express the general level of neuropsychological performance. As an index of effect size in the parametric statistical tests we report Eta-squared $\left(\eta^{2}\right)$, which can vary between 0 and 1 .

\section{RESULTS}

Data from 146 consecutive MCI subjects of primary aetiology together with data from 50 gender matched controls were analyzed. The control group, ( $65 \pm 6$ years) was significantly older than the MCI group (62 \pm 7 years), $p=$ .035. There was no difference as to gender distribution; both groups consisted of $46 \%$ males. The controls scored significantly higher on MMSE, $29.3 \pm 1.0$, than the MCI group, $28.5 \pm 1.3(p<.001)$. There was no significant difference as to formal education: controls $11.4 \pm 2.4$ years, MCI $12.0 \pm 3.4$ years.

The CSF total tau of the control group was $291 \pm 102$ $\mathrm{pg} / \mathrm{mL}$, which was significantly lower than in the MCI group, $416 \pm 328 \mathrm{pg} / \mathrm{mL}(p<.001)$. The CSF A $\beta 42$ of the control group was $750 \pm 224 \mathrm{pg} / \mathrm{mL}$, which was significantly higher than in the MCI group, $622 \pm 197 \mathrm{pg} / \mathrm{mL}(p=.001)$. Using the 0.90 fractile of the control group values as a cut off, (Chemistry, 1987) $40 \%$ of the MCI subjects had high T-tau concentrations $(>405 \mathrm{pg} / \mathrm{mL})$ and $26 \%$ low $\mathrm{A} \beta 42$ concentrations $(<465 \mathrm{pg} / \mathrm{mL})$. Seventy-three (50\%) of the consecutive MCI subjects of primary aetiology were found to have normal concentrations of CSF T-tau and A $\beta 42$ (MCI-norm). There was considerable overlap between the subjects with elevated T-tau 
and low $\mathrm{A} \beta 42 ; 23$ subjects had high T-tau and low $\mathrm{A} \beta 42$ concentrations, 35 had only high T-tau and 15 only low $\mathrm{A} \beta 42$, as seen in Fig. 1.

The subjects were also genotyped for Apolipoprotein E (APOE), which has 3 major alleles, APOE2, APOE3, and APOE4. APOE4 is a known risk factor not only for $\mathrm{AD}$, but also for generally impaired cognitive function and atherosclerosis (Stojakovic et al., 2004; Wehling et al., 2007). The groups differed significantly in terms of incidence of APOE4: $22(30 \%)$ of the MCI-norm subjects had an APOE4 allele, whereas $42(57 \%)$ of those with AD-typical biomarkers had an APOE4 allele $(p=.001)$.

We began our analyses by comparing the subjects with deviating results in CSF (MCI-dev) to those with normal results (MCI-norm) and controls. As seen in Table 2, the groups did not differ regarding general intellectual capacity, as assessed with "Raven's Coloured Matrices." The MCInorm group was significantly younger and better educated, whereas controls and MCI-dev did not differ on those variables.

The weighted average (PCA) score indicates that the controls performed overall better than MCI-norm, who in turn performed better than MCI-dev. After adjustment for multiple comparisons there were significant differences between controls and MCI-norm on 7 of the 22 neuropsychological test variables; controls performed better on one speed and attention, one visuospatial, 2 language, and 3 executive tests. The differences between controls and MCI-

Table 2. Means and significance levels for demographic data and neuropsychology

\begin{tabular}{|c|c|c|c|c|c|c|c|}
\hline Demographic data & $\begin{array}{l}\text { Controls } \\
(N=50)\end{array}$ & $\begin{array}{c}\text { MCI-norm } \\
(N=73)\end{array}$ & $\begin{array}{l}\text { MCI-dev } \\
(N=73)\end{array}$ & Eta2 & $\begin{array}{c}\text { Controls } \\
\text { Versus } \\
\text { MCI-norm } \\
\text { adjusted } p\end{array}$ & $\begin{array}{l}\text { Controls } \\
\text { Versus } \\
\text { MCI-dev } \\
\text { adjusted } p\end{array}$ & $\begin{array}{l}\text { MCI-norm } \\
\text { Versus } \\
\text { MCI-dev } \\
\text { adjusted } p\end{array}$ \\
\hline Age & $65.1 \pm 6.1$ & $60.7 \pm 6.8$ & $64.6 \pm 7.5$ & & .002 & .967 & .002 \\
\hline Gender (male/female) & $23 / 27$ & $35 / 38$ & $31 / 42$ & & ns & ns & $\mathrm{ns}$ \\
\hline Education & $11.4 \pm 2.4$ & $12.8 \pm 3.3$ & $11.3 \pm 3.4$ & & .040 & .999 & .012 \\
\hline MMSE & $29.3 \pm 1.0$ & $28.6 \pm 1.3$ & $28.3 \pm 1.2$ & & .012 & $<.001$ & .194 \\
\hline \multicolumn{8}{|l|}{ Neuropsychological data } \\
\hline Weighted average & 1.56 & .88 & -2.13 & .22 & .006 & $<.001$ & $<.001$ \\
\hline \multicolumn{8}{|l|}{ Speed and attention } \\
\hline Digit Span & $13.8 \pm 3.5$ & $13.6 \pm 3.3$ & $13.1 \pm 3.2$ & .01 & .967 & .637 & .876 \\
\hline Digit Symbol & $47.5 \pm 9.8$ & $46.5 \pm 11.0$ & $39.2 \pm 10.9$ & .11 & .204 & $<.001 *$ & $.012 *$ \\
\hline Trail Making A & $35.6 \pm 11.3$ & $38.4 \pm 13.3$ & $46.4 \pm 17.7$ & .12 & $.016^{*}$ & $<.001 *$ & .054 \\
\hline Trail Making B & $81.8 \pm 27.5$ & $87.8 \pm 34 . .9$ & $114.5 \pm 48.6$ & .12 & .102 & $<.001 *$ & $.012 *$ \\
\hline \multicolumn{8}{|l|}{ Memory and learning } \\
\hline RAVLT learning & $44.5 \pm 7.9$ & $45.8 \pm 8.6$ & $37.2 \pm 11.5$ & .12 & .995 & $<.001 *$ & $<.001 *$ \\
\hline RAVLT delayed recall & $8.8 \pm 3.0$ & $8.7 \pm 2.9$ & $6.2 \pm 4.0$ & .10 & .858 & $<.001 *$ & $.001 *$ \\
\hline WLM delayed recall & $21.1 \pm 6.0$ & $21.1 \pm 7.0$ & $16.2 \pm 10.3$ & .04 & .978 & .090 & .173 \\
\hline RCF delayed recall & $16.6 \pm 6.0$ & $16.5 \pm 7.5$ & $10.3 \pm 7.1$ & .14 & .488 & $<.001 *$ & $<.001^{*}$ \\
\hline RAVLT recognition & $14.7 \pm 0.5$ & $14.6 \pm 0.8$ & $13.9 \pm 1.8$ & .08 & .851 & $.001 *$ & $.007^{*}$ \\
\hline \multicolumn{8}{|l|}{ Visuospatial functions } \\
\hline VOSP Silhouettes & $22.3 \pm 3.0$ & $21.2 \pm 4.5$ & $18.7 \pm 4.7$ & .08 & $.030 *$ & $<.001 *$ & .105 \\
\hline RCF copying & $32.1 \pm 2.6$ & $31.5 \pm 5.5$ & $30.4 \pm 5.4$ & .01 & .949 & .660 & .923 \\
\hline Block Design & $29.9 \pm 8.2$ & $29.9 \pm 8.9$ & $25.9 \pm 8.6$ & .04 & .531 & $.030 *$ & .329 \\
\hline \multicolumn{8}{|l|}{ Language } \\
\hline Token Test & $21.1 \pm 1.2$ & $20.1 \pm 1.7$ & $18.8 \pm 2.9$ & .16 & $.001 *$ & $<.001 *$ & .060 \\
\hline Boston Naming Test & $55.3 \pm 2.9$ & $53.5 \pm 5.2$ & $50.2 \pm 6.9$ & .14 & $.007 *$ & $<.001 *$ & $.050 *$ \\
\hline ASLD Repetition & $21.1 \pm 5.2$ & $19.4 \pm 6.0$ & $17.4 \pm 6.6$ & .06 & .150 & $.005 *$ & .531 \\
\hline Similarities & $21.1 \pm 2.9$ & $21.1 \pm 3.4$ & $19.4 \pm 4.0$ & .03 & .723 & $.028 *$ & .227 \\
\hline Word Fluency FAS & $42.7 \pm 13.5$ & $42.4 \pm 11.6$ & $37.9 \pm 12.5$ & .02 & .877 & .084 & .303 \\
\hline \multicolumn{8}{|l|}{ Executive functions } \\
\hline PaSMO & $64.9 \pm 20.8$ & $73.4 \pm 24.8$ & $88.9 \pm 39.6$ & .10 & $.036^{*}$ & $<.001 *$ & 0.297 \\
\hline Dual Task & $52.1 \pm 8.7$ & $49.9 \pm 10.0$ & $48.4 \pm 11.4$ & .03 & .152 & .122 & 0.997 \\
\hline Stroop & $26.3 \pm 6.6$ & $29.8 \pm 10.7$ & $34.6 \pm 12.7$ & .10 & $.033^{*}$ & $.001 *$ & 0.407 \\
\hline WCST-CV64 & $44.3 \pm 8.4$ & $39.6 \pm 14.0$ & $35.3 \pm 14.9$ & .07 & $.031 *$ & $.020 *$ & 0.953 \\
\hline Cognitive Estimation & $3.0 \pm 1.5$ & $3.6 \pm 1.9$ & $4.4 \pm 2.0$ & .08 & .090 & $.002 *$ & 0.348 \\
\hline
\end{tabular}

MCI-norm $=$ MCI with normal concentrations of CSF T-tau and A $\beta 42, \mathrm{MCI}-\mathrm{dev}=\mathrm{MCI}$ with deviating concentrations of CSF T-tau and/or A $\beta 42, *=$ mean difference is significant on 0.05 level, WAIS-R = Wechler's Adult Intelligence Scale-Revised, RAVLT $=$ Rey Auditory Verbal Learning Test, WMS-R = Wechler's Memory Scale-Revised, VOSP = Visual Object and Space Perception, ASLD = Assessment of Subtle Language Deficits, PaSMO = Parallell Serial Mental Operations, WCST-CV64 = Wisconsin Card Sorting Test-Computer Version 64 (short version). 
dev were pronounced-controls performed significantly better on 17 test variables; 3 speed and attention tests, 4 episodic memory variables, 2 visuospatial, 4 language, and 4 executive tests. The most clear-cut differences-with the highest significance levels and Eta-squared scores-were recorded on the Trail Making tests, RAVLT and RCF delayed recall, Token Test, and Boston Naming Test. On seven test variables MCI-norm performed significantly better than MCI-dev; 2 speed and attention tests, 4 episodic memory variables, and one language test. The most clearcut differences were on the episodic memory variables.

We continued our analyses by grouping the MCI-dev subjects into three groups, one with only high T-tau (MCI-tau), $N=35$, one with only low $\mathrm{A} \beta 42$ (MCI-A $\beta$ ), $N=15$, and the last with both high T-tau and low $\mathrm{A} \beta 42$ (MCI-tauA $\beta$ ), $N=23$. MCI-tauA $\beta$ tended to perform slightly worse than the two other groups, but there were few statistically significant differences after adjustment for multiple comparisons. MCI-tau performed significantly better than MCI-A $\beta$ on a language test, and MCI-tau performed significantly better than MCI-tauA $\beta$ on 3 tests; one speed and attention, one memory, and one language test, as seen in Table 3.

We further continued the analyses by subclassifying the 146 MCI subjects into the four types of MCI suggested by Petersen in 2004. We did this by setting a cut off for each test at 1.5 standard deviations below the mean of age appropriate controls, in order to establish a level of low performance for age, and thus approximate the prevalent MCI criteria. We then calculated the proportion of subjects exhibiting impairment on one or more tests within each cognitive domain. As seen in Table 4, the most common MCI subtype was the multidomain amnestic (memory impairment and other cognitive impairment), followed by multidomain nonamnestic. The rarest subtype was the amnestic, and 20 subjects $(14 \%)$ did not exhibit any significant impairment as compared to age norms.
Table 4 also shows that the majority of the two multidomain subtype MCI subjects, 59\%, had high T-tau and/or low $\mathrm{A} \beta 42$ concentrations, whereas only $33 \%$ of the amnestic and $28 \%$ of the single domain non-memory groups had deviating concentrations. In fact, those proportions were smaller than the proportion in the "no impairment" group, $50 \%$.

\section{DISCUSSION}

Our objective was to compare the neuropsychological profiles of MCI subjects with normal concentrations of T-tau and $\mathrm{A} \beta 42$ in CSF to the profiles of MCI subjects with high concentrations of T-tau and low concentrations of $\mathrm{A} \beta 42$. We found significant overall neuropsychological differences between controls and both MCI groups. Further, we found significant differences between MCI subjects with normal concentrations of the biomarkers (MCI-norm) and those with high T-tau and/or low A $\beta 42$ (MCI-dev), most clearly on tests of episodic memory and speed/attention. This would suggest that deficits in these cognitive domains are the most crucial deficits in MCI subjects who probably are in the prodromal stages of AD.

When MCI-norm, MCI subjects without vascular disease and biomarkers associated with $\mathrm{AD}$ were compared to healthy controls, the neuropsychological differencesalthough some significant-were strikingly small. On memory tests the results were almost identical, and on the speed and attention tests very similar. On two language tests, however, there were highly significant differences and on three executive tests significant differences. On the language and executive tests a small number of MCI-norm subjects performed poorly. One possible explanation is that poor performance on language tests was because of prodromal Primary Progressive Aphasia or Semantic Dementia, and on the executive tests because of prodromal frontotemporal

Table 3. MCI groups with high $\mathrm{T}-\tau$ (MCI- $\tau)$, low $\mathrm{A} \beta 42$ (MCI-A $\beta$ ), and both high T-tau and low A $\beta 42(\mathrm{MCI}-\tau \mathrm{A} \beta$ ) in CSF Means and significance levels for demographic data

\begin{tabular}{|c|c|c|c|c|c|c|c|}
\hline Demographic data & $\begin{array}{c}\mathrm{MCI}-\tau \\
(N=35)\end{array}$ & $\begin{array}{l}\mathrm{MCI}-\mathrm{A} \beta \\
(N=15)\end{array}$ & $\begin{array}{c}\mathrm{MCI}-\tau \mathrm{A} \beta \\
(N=23)\end{array}$ & Eta2 & $\begin{array}{c}\text { MCI- } \tau \\
\text { Versus } \\
\text { MCI-A } \beta \\
\text { adjusted } p\end{array}$ & $\begin{array}{c}\text { MCI- } \tau \\
\text { Versus } \\
\text { MCI- } \tau \mathrm{A} \beta \\
\text { adjusted } p\end{array}$ & $\begin{array}{c}\text { MCI-A } \beta \\
\text { Versus } \\
\text { MCI- } \tau \mathrm{A} \beta \\
\text { adjusted } p\end{array}$ \\
\hline Age & $64.1 \pm 6.9$ & $62.7 \pm 7.7$ & $66.6 \pm 7.9$ & & .908 & .517 & .321 \\
\hline Gender (male/female) & $16 / 19$ & $7 / 8$ & $9 / 14$ & & ns & ns & ns \\
\hline Education & $11.7 \pm 3.5$ & $11.6 \pm 2.9$ & $10.5 \pm 3.4$ & & .998 & .397 & .681 \\
\hline MMSE & $28.6 \pm 1.0$ & $28.6 \pm 1.3$ & $27.8 \pm 1.4$ & & 1.000 & $.040 *$ & .108 \\
\hline Raven's Coloured Matrices & $32.4 \pm 2.6$ & $32.6 \pm 1.9$ & $31.1 \pm 3.5$ & & .999 & .098 & .101 \\
\hline \multicolumn{8}{|l|}{ Neuropsychological data } \\
\hline Weighted average (PCA) & -1.38 & -1.33 & -3.78 & .05 & .975 & .190 & .341 \\
\hline Trail Making B & $104.8 \pm 42.3$ & $98.1 \pm 29 . .0$ & $139.9 \pm 58.5$ & .10 & .999 & $.045^{*}$ & .102 \\
\hline WLM delayed recall & $19.0 \pm 11.0$ & $19.1 \pm 7.5$ & $10.8 \pm 8.8$ & .14 & .922 & $.019 *$ & .220 \\
\hline ASLD Repetition & $20.3 \pm 5.4$ & $14.9 \pm 7.7$ & $15.4 \pm 6.1$ & .15 & $.036^{*}$ & $.030 *$ & .998 \\
\hline
\end{tabular}

MCI-tau $=$ MCI with high concentrations of CSF T-tau, MCI-A $\beta=$ MCI with low concentrations of CSF A $\beta 42$, MCI-tauA $\beta=$ MCI with high concentrations of CSF T-tau and low of A $\beta 42$, PCA = Principal Component Analysis, ${ }^{*}=$ mean difference is significant on 0.05 level, WLM $=$ Wechsler's Logical Memory, ASLD = Assessment of Subtle Language Deficits. 
Table 4. Classification by MCI subtype

\begin{tabular}{lcrrrr}
\hline \hline MCI subtype & Number of MCI & MCI-norm & MCI- $\tau$ & MCI-A $\beta$ & MCI- $\tau$ A $\beta$ \\
\hline Amnestic & 6 & $4(66 \%)$ & $1(17 \%)$ & $1(17 \%)$ & \multicolumn{1}{c}{0} \\
Multidomain amnestic & 49 & $18(37 \%)$ & $15(30 \%)$ & $5(10 \%)$ & $11(23 \%)$ \\
Multidomain non-amnestic & 41 & $19(46 \%)$ & $9(22 \%)$ & $5(12 \%)$ & $8(20 \%)$ \\
Single domain non-memory & 30 & $22(73 \%)$ & $5(17 \%)$ & $2(7 \%)$ & $1(3 \%)$ \\
No impairment & 20 & $10(50 \%)$ & $5(25 \%)$ & $2(10 \%)$ & $3(15 \%)$ \\
\hline \hline
\end{tabular}

or Lewy Body dementia. Most subjects also experienced stress in their every day lives. Were they stressed because of poor cognitive function, or did they perform poorly due to stress? Stress is known to cause executive problems, thus some of the executive symptoms in the MCI-norm group may have been caused by stress. Still it would seem that the majority of MCI subjects who had no signs of vascular or biochemical abnormalities showed very mild cognitive deficits and performed more like healthy persons. This was also illustrated by the weighted average (PCA) composite score, on which the difference between controls and MCInorm was clearly smaller than between MCI-norm and MCI-dev.

The fact that almost all MCI subjects attended our clinic because of memory complaints makes the results of the MCI-norm group surprising; a large proportion of the subjects with memory complaints performed within the normal range on episodic memory tests. The results from the subclassification by MCI type also illustrate this: a minority, $38 \%$, belonged to the amnestic groups and the purely amnestic group was small. One possible explanation for this is that the memory tests lack ecological validity (i.e., they do not measure the kind of memory trouble the subjects experience in their everyday lives). In fact, many subjects described memory problems, which could be characterized as prospective memory problems; remembering what to do in the future rather than remembering the past. Prospective memory is considered to have a distinct executive component (Fish et al., 2007), which would agree with the differences between controls and MCI-norm on the executive tests. Another explanation could be that many subjects think of language (naming) problems as trouble remembering words (i.e., memory problems).

When the MCI-dev group is compared to controls, it appears quite heterogeneous, there are significant differences in all cognitive domains. The differences in memory performance are no more pronounced than the differences in the language or speed and attention domains. If we are to assume that a majority of these subjects are in the preclinical stages of $\mathrm{AD}$, the results support the notion that $\mathrm{AD}$ is preceded by impairment in multiple cognitive domains.

The finding that the most clear-cut differences between MCI-norm and MCI-dev were on the episodic memory tests is perhaps not surprising, considering that previous studies have found high T-tau to be associated with poor memory performance (Ivanoiu \& Sindic, 2005; Schoonenboom et al.,
2005). The MCI-dev group also consisted of subjects with low $\mathrm{A} \beta 42$, which would make the differences in the speed/ attention and language domains in accordance with previous studies, in which low $\mathrm{A} \beta 42$ has been associated with poorer general cognitive function (Ivanoiu \& Sindic, 2005; Schoonenboom et al., 2005). This distinction could, however, not be seen when the CSF group was subclassified into MCI-tau and MCI-A $\beta$, the memory test scores were almost identical. Interestingly, MCI-tauA $\beta$ performed somewhat worse on the memory tests, on WLM significantly so. It would seem that not one or the other of the biomarkers, but the biomarkers combined, are associated with markedly poor memory performance.

Twenty MCI subjects did not show any cognitive impairment as compared to their age norm. Some of these subjects may well be "healthy worried," but the main explanation probably is that they are better educated ( $M=14.5$ years) and consist of a number of subjects with high premorbid cognitive capacity. They experienced cognitive decline but because of their superior capacity were able to compensate for the decline, and still performed at an average level for their age. This is a hypothesis that has been put forward previously (Nordlund et al., 2005; Schmand et al., 1997). The fact that 10 of these subjects had either high T-tau or low $\mathrm{A} \beta 42$ concentrations, which indicates degenerative processes in the brain, supports this explanation. These findings, once again, raise the question about the validity of the MCI concept - should the diagnosis of MCI not be based on an assessment of premorbid capacity (Rentz et al., 2004)?

It may not be considered surprising that two biomarkers associated with $\mathrm{AD}$ also are associated with poor cognitive performance in MCI. One novel finding of this study, however, is that on a comprehensive neuropsychological battery there were hardly any differences between subjects with high $\mathrm{T}$-tau and low $\mathrm{A} \beta 42$. It seems that in MCI both biomarkers are associated with marked general cognitive impairment when compared to MCI subjects with normal biomarker concentrations, and when both markers are present, the cognitive impairment is even more pronounced.

One interesting future avenue for early diagnostics is examining the effect of the combination of CSF biomarkers and APOE4 on cognition. The proportion of APOE4 carriers in the MCI-dev group was almost twice the proportion in the MCI-norm group. APOE4 is a risk factor not only for $\mathrm{AD}$, but for poor cognition in general and the CSF biomarkers are markers for degenerative processes in the brain. That 
combination certainly is interesting to link to the results on a neuropsychological battery. In fact, work on such an article is already well in progress.

One recurring finding in studies on $\mathrm{MCI}$ is that a considerable proportion of MCI subjects have "benign" forms of MCI; they either are stationary (i.e., do not progress to dementia) or even improve over time (Bozoki et al., 2001; Guarch et al., 2004; Ritchie et al., 2001). More exact diagnostic procedures have been called for (Luis et al., 2003), and are obviously needed in order to, as early as possible, identify the subjects who are at greatest risk for dementia. This task is urgent but obviously complicated. Considering the neuropsychological differences we have presentedparticularly on the episodic memory tests, between MCInorm and MCI-dev, we believe that many subjects in the MCI-norm group have more benign forms of MCI, or early non-AD forms of neurodegenerative disorders. Although most MCI-dev subjects performed clearly worse than MCInorm on the neuropsychological battery, some did not show any deficits when compared to age norms. In these cases thorough neuropsychological considerations and perhaps tests with better ecological validity are needed. Thus, the combination of CSF analyses and a comprehensive neuropsychological assessment, taking into consideration premorbid capacity, could be a step toward a more exact differential diagnosis of MCI as preliminary AD.

\section{ACKNOWLEDGMENTS}

The authors thank research nurses Christina Holmberg and Ewa Styrud for their invaluable work in monitoring the study. This work was supported by grants from Alzheimerfonden; Axel Linders Stiftelse; Pfannenstills stiftelse; Stiftelsen för Gamla Tjänarinnor; Stiftelsen Hjalmar Svenssons forskningsfond.

\section{REFERENCES}

AAN. (1996). Assessment: Neuropsychological testing of adults. Considerations for neurologists. Report of the Therapeutics and Technology Assessment Subcommittee of the American Academy of Neurology. Neurology, 47, 592-599.

Albert, M.S., Moss, M.B., Tanzi, R., \& Jones, K. (2001). Preclinical prediction of AD using neuropsychological tests. Journal of the International Neuropsychological Society, 7, 631-639.

Andreasen, N., Hesse, C., Davidsson, P., Minthon, L., Wallin, A., Winblad, B., Vanderstichele, H., Vanmechelen, E., \& Blennow, K. (1999). Cerebrospinal fluid beta-amyloid(1-42) in Alzheimer disease: Differences between early- and late-onset Alzheimer disease and stability during the course of disease. Archives of Neurology, 56, 673-680.

Andreasen, N., Vanmechelen, E., Vanderstichele, H., Davidsson, P., \& Blennow, K. (2003). Cerebrospinal fluid levels of totaltau, phospho-tau and A beta 42 predicts development of Alzheimer's disease in patients with mild cognitive impairment. Acta Neurologica Scandinavica Suppl., 179, 47-51.

Binetti, G., Cappa, S.F., Magni, E., Padovani, A., Bianchetti, A., \& Trabucchi, M. (1996). Disorders of visual and spatial per- ception in the early stage of Alzheimer's disease. Annals of the New York Academy of Science, 777, 221-225.

Blackwell, A.D., Sahakian, B.J., Vesey, R., Semple, J.M., Robbins, T.W., \& Hodges, J.R. (2004). Detecting dementia: Novel neuropsychological markers of preclinical Alzheimer's disease. Dementia in Geriatric Cognitive Disorder, 17, 42-48.

Blennow, K. (2004a). Cerebrospinal fluid protein biomarkers for Alzheimer's disease. NeuroRx, 1, 213-225.

Blennow, K. (2004b). CSF biomarkers for mild cognitive impairment. Journal of Internal Medicine, 256, 224-234.

Blennow, K., Wallin, A., Agren, H., Spenger, C., Siegfried, J., \& Vanmechelen, E. (1995). Tau protein in cerebrospinal fluid: A biochemical marker for axonal degeneration in Alzheimer disease? Molecular Chemistry and Neuropathology, 26, 231-245.

Bozoki, A., Giordani, B., Heidebrink, J.L., Berent, S., \& Foster, N.L. (2001). Mild cognitive impairments predict dementia in nondemented elderly patients with memory loss. Archives of Neurology, 58, 411-416.

Busse, A., Hensel, A., Guhne, U., Angermeyer, M.C., \& RiedelHeller, S.G. (2006). Mild cognitive impairment: Long-term course of four clinical subtypes. Neurology, 67, 2176-2185.

Chemistry, I.F.o.C. (1987). Approved recommendation on the theory of reference values. Part 5. Statistical treatment of collected reference values. Determination of reference limits. Clinical Chimica Acta, 170, 13-32.

Eklund, G. \& Seeger, P. (1965). Massignifikans-analys. Statistisk Tidskrift, 3, 355-365.

Eriksson, L., Johansson, E., Kettaneh-Wold, N., Wikström, C., \& Wold, S. (2002). User's Guide to Simca-P. Umea, Sweden: Umetrics AB.

Fish, J., Evans, J.J., Nimmo, M., Martin, E., Kersel, D., Bateman, A., Wilson, B.A., \& Manly, T. (2007). Rehabilitation of executive dysfunction following brain injury: "Content-free" cueing improves everyday prospective memory performance. Neuropsychologia, 45, 1318-1330.

Folstein, M.F., Folstein, S.E., \& McHugh, P.R. (1975). "Minimental state". A practical method for grading the cognitive state of patients for the clinician. Journal of Psychiatric Research, 12, 189-198.

Grundman, M., Petersen, R.C., Ferris, S.H., Thomas, R.G., Aisen, P.S., Bennett, D.A., Foster, N.L., Jack, C.R., Jr., Galasko, D.R., Doody, R., Kaye, J., Sano, M., Mohs, R., Gauthier, S., Kim, H.T., Jin, S., Schultz, A.N., Schafer, K., Mulnard, R., van Dyck, C.H., Mintzer, J., Zamrini, E.Y., Cahn-Weiner, D., \& Thal, L.J. (2004). Mild cognitive impairment can be distinguished from Alzheimer disease and normal aging for clinical trials. Archives of Neurology, 61, 59-66.

Guarch, J., Marcos, T., Salamero, M., \& Blesa, R. (2004). Neuropsychological markers of dementia in patients with memory complaints. International Journal of Geriatric Psychiatry, 19, 352-358.

Hansson, O., Zetterberg, H., Buchhave, P., Londos, E., Blennow, K., \& Minthon, L. (2006). Association between CSF biomarkers and incipient Alzheimer's disease in patients with mild cognitive impairment: A follow-up study. Lancet Neurology, 5 , $228-234$

Ivanoiu, A. \& Sindic, C.J. (2005). Cerebrospinal fluid TAU protein and amyloid beta42 in mild cognitive impairment: Prediction of progression to Alzheimer's disease and correlation with the neuropsychological examination. Neurocase, 11, $32-39$. 
Luis, C.A., Loewenstein, D.A., Acevedo, A., Barker, W.W., \& Duara, R. (2003). Mild cognitive impairment: Directions for future research. Neurology, 61, 438-444.

Morris, J.C. (1997). Clinical dementia rating: A reliable and valid diagnostic and staging measure for dementia of the Alzheimer type. International Psychogeriatrics, 9 (Suppl 1), 173-176 (discussion 177-178).

Morris, J.C., Storandt, M., Miller, J.P., McKeel, D.W., Price, J.L., Rubin, E.H., \& Berg, L. (2001). Mild cognitive impairment represents early-stage Alzheimer disease. Archives of Neurology, 58, 397-405.

Nordlund, A., Rolstad, S., Hellstrom, P., Sjogren, M., Hansen, S., \& Wallin, A. (2005). The Goteborg MCI study: Mild cognitive impairment is a heterogeneous condition. Journal of Neurology, Neurosurgery and Psychiatry, 76, 1485-1490.

Petersen, R.C. (2000). Mild cognitive impairment: Transition between aging and Alzheimer's disease. Neurologia, 15, 93-101.

Petersen, R.C. (2004). Mild cognitive impairment as a diagnostic entity. Journal of Internal Medicine, 256, 183-194.

Petersen, R.C., Smith, G.E., Waring, S.C., Ivnik, R.J., Tangalos, E.G., \& Kokmen, E. (1999). Mild cognitive impairment: Clinical characterization and outcome. Archives of Neurology, 56, 303-308.

Rapport, L.J., Millis, S.R., \& Bonello, P.J. (1998). Validation of the Warrington theory of visual processing and the Visual Object and Space Perception Battery. Journal of Clinical and Experimental Neuropsychology, 20, 211-220.

Rasquin, S.M., Lodder, J., Visser, P.J., Lousberg, R., \& Verhey, F.R. (2004). Predictive Accuracy of MCI Subtypes for Alzheimer's Disease and Vascular Dementia in Subjects with Mild Cognitive Impairment: A 2-Year Follow-Up Study. Dementia and Geriatric Cognitive Disorders, 19, 113-119.

Rentz, D.M., Huh, T.J., Faust, R.R., Budson, A.E., Scinto, L.F., Sperling, R.A., \& Daffner, K.R. (2004). Use of IQ-adjusted norms to predict progressive cognitive decline in highly intelligent older individuals. Neuropsychology, 18, 38-49.

Ritchie, K., Artero, S., \& Touchon, J. (2001). Classification criteria for mild cognitive impairment: A population-based validation study. Neurology, 56, 37-42.

Royall, D.R., Mahurin, R.K., \& Gray, K.F. (1992). Bedside assessment of executive cognitive impairment: The executive interview. Journal of the American Geriatric Society, 40, 1221-1226.

Scheltens, P., Erkinjunti, T., Leys, D., Wahlund, L.O., Inzitari, D., del Ser, T., Pasquier, F., Barkhof, F., Mantyla, R., Bowler, J.,
Wallin, A., Ghika, J., Fazekas, F., \& Pantoni, L. (1998). White matter changes on CT and MRI: An overview of visual rating scales. European Task Force on Age-Related White Matter Changes. European Neurology, 39, 80-89.

Schmand, B., Smit, J.H., Geerlings, M.I., \& Lindeboom, J. (1997). The effects of intelligence and education on the development of dementia. A test of the brain reserve hypothesis. Psychological Medicine, 27, 1337-1344.

Schoonenboom, S.N., Visser, P.J., Mulder, C., Lindeboom, J., Van Elk, E.J., Van Kamp, G.J., \& Scheltens, P.H. (2005). Biomarker profiles and their relation to clinical variables in mild cognitive impairment. Neurocase, 11, 8-13.

Stojakovic, T., Scharnagl, H., \& Marz, W. (2004). ApoE: Crossroads between Alzheimer's disease and atherosclerosis. Seminars in Vascular Medicine, 4, 279-285.

Storandt, M., Grant, E.A., Miller, J.P., \& Morris, J.C. (2002). Rates of progression in mild cognitive impairment and early Alzheimer's disease. Neurology, 59, 1034-1041.

Testa, J.A., Ivnik, R.J., Boeve, B., Petersen, R.C., Pankratz, V.S., Knopman, D., Tangalos, E., \& Smith, G.E. (2004). Confrontation naming does not add incremental diagnostic utility in $\mathrm{MCI}$ and Alzheimer's disease. Journal of the International Neuropsychological Society, 10, 504-512.

Wallin, A., Edman, A., Blennow, K., Gottfries, C.G., Karlsson, I., Regland, B., \& Sjogren, M. (1996). Stepwise comparative status analysis (STEP): A tool for identification of regional brain syndromes in dementia. Journal of Geriatric Psychiatry and Neurology, 9, 185-199.

Wehling, E., Lundervold, A.J., Standnes, B., Gjerstad, L., \& Reinvang, I. (2007). APOE status and its association to learning and memory performance in middle aged and older Norwegians seeking assessment for memory deficits. Behavioral Brain Functions, 3, 57.

Winblad, B., Palmer, K., Kivipelto, M., Jelic, V., Fratiglioni, L., Wahlund, L.O., Nordberg, A., Backman, L., Albert, M., Almkvist, O., Arai, H., Basun, H., Blennow, K., de Leon, M., DeCarli, C., Erkinjuntti, T., Giacobini, E., Graff, C., Hardy, J., Jack, C., Jorm, A., Ritchie, K., van Duijn, C., Visser, P., \& Petersen, R.C. (2004). Mild cognitive impairment-beyond controversies, towards a consensus: Report of the International Working Group on Mild Cognitive Impairment. Journal of Internal Medicine, 256, 240-246. 